\title{
Considering the effects of a RTV coating to improve electrical insulation against lightning
}

\begin{abstract}
One of the factors that cause power outages on distribution lines is being struck by lightning. Cloud to ground lightning often affects overhead distribution lines and can occur with negative or positive polarities. It can affect the performance of insulators and cause power line failure. Furthermore, outdoor polymer insulators are exposed to extreme weathering and pollution, which can cause chemical changes in the material properties and further leads to material degradation. These can cause a reduction in the insulator withstand capabilities and may lead to flashover. This paper investigates the effects of a RTV (room temperature vulcanisation) coating on a $10 \mathrm{kV}$ polymer insulator in order to improve the electrical insulation performance against a lightning impulse under clean and polluted conditions. The up and down test method was used to evaluate the withstand voltage capabilities of an RTV coated polymer insulator. For reference, the withstand voltage for a basic setting polymer insulator was measured for comparison.
\end{abstract}

Keyword: Lightning; Component; Polymer insulator; RTV coating; Withstand voltage 